the lungs include oedema, haemorrhage, and hyaline membrane formation. If, however, the affected animals are removed from the highly oxygenated environment and are returned to air they may recover completely. Oxygen therapy is thus a subject to be approached with some caution. ${ }^{12}$ A team of experts should be available to monitor the oxygen content of alveolar air and of the arterial blood in order that dangerously high concentrations may be avoided. Safe administration of oxygen also requires the judgement of the clinician on whether an infant may not be returned to air, at least for a period, without too great a risk of tissue anoxia.

\section{Coal Gas and the Brain}

The common methods of attempting suicide in Britain are inhalation of carbon monoxide and ingestion of barbiturates and aspirin. Accidental exposure to coal gas adds to the number of cases of carbon monoxide poisoning, so that each year it rises to several thousand. As the affinity of haemoglobin for carbon monoxide approaches 300 times that which it has for oxygen, and as nervous tissue is peculiarly and rapidly sensitive to oxygen lack, the major brunt of the toxic symptoms is borne by the central nervous system. In rapidly fatal cases of carbon monoxide poisoning the findings at necropsy are congestion and haemorrhages in all the organs, and these show the characteristic pink colour; but in less acutely fatal cases ischaemic changes, though seen, are less dramatic in other organs than are the anoxic and ischaemic lesions in the brain. Here there is necrosis in the second and third layers of the cortex and the superficial white matter, with degeneration of the Purkinje cells in the cerebellum and the cells of Sommer's sector of Ammon's horn. ${ }^{1}$ When death occurs later still there is extensive demyelination with gliosis and cerebral atrophy. Lesions may be found at all stages in the globus pallidus, and these are probably of vascular origin.

As the changes are widespread and diffuse, it is not surprising that the symptoms and sequelae of carbon monoxide intoxication cover almost the whole spectrum of cerebral dysfunction. What is surprising, however, is that patients who survive the acute stage only rarely suffer permanent effects. Death is common $(20-40 \%)$, but if the patient lives recovery is usually complete. In a survey in New York State of over 21,100 cases of carbon monoxide poisoning only 39 patients were found to have permanent sequelae. ${ }^{2}$

Early symptoms are also very varied. Euphoria, lack of judgement, headache, tightness across the forehead, and blurring of vision may all occur, progressing to confusion, disorientation, ataxia, drowsiness, and coma. Apart from the headache and visual symptoms the patient may be unaware of these features. If he is also unaware of the gas he may reach a state in which muscle anoxia prevents him from withdrawing from the danger even if consciousness is not lost early, as it will be if the patient is suddenly subjected to high concentrations. This is sometimes the fate of gallant would-be rescuers who have not paused to consider the possible cause of unconsciousness in a victim lying in an enclosed space.

Meyer, A., in Greenfield's Neuropathology, 2nd ed., 1963. London.

- Shillito, F. H., Drinker, C. K., and Shaughnessy, T. J., f. Amer. med. Ass., 1936, 106, 669.

- Smith, G., Ledingham, I. McA., Sharp, G. R., Norman, J. N., and Bates, E. H., Lancet, 1962, 1, 816.

- Garland, H., and Pearce, J., Quart. Ұ. Med., 1967, 36, 445

- Garland, H., and Prit. med. Ұ., 1965, $1,1232$.

- Plum, F., Posner, J. B., and Hain, R. F., Arch. intern. Med., 1962, 110,18 .
Treatment must be directed at rapid removal of the patient from the source of the gas, and then conversion of the carboxyhaemoglobin to oxyhaemoglobin. Pure oxygen or oxygen with $5-7 \%$ carbon dioxide should be given as quickly as possible, and in comatose patients the use of intravenous hypertonic solutions may serve to reduce cerebral oedema. However, where this is available, exposure to hyperbaric oxygen at $2-2 \frac{1}{2}$ atmospheres pressure is the treatment of choice, and in a series of 22 cases treated in this way in Glasgow a $100 \%$ recovery rate with no sequelae was obtained. ${ }^{3}$

The sequelae of carbon monoxide poisoning can vary from permanent confusion and disorientation to merely a generalized increase in tendon reflexes, or conversely signs of a peripheral neuropathy. All degrees of Parkinsonism and dystonia have also been recorded, though the frequency of this complication has probably been overemphasized. The physiology, pathology, and clinical manifestations of carbon monoxide poisoning as it affects the nervous system have been reviewed recently by the late Hugh Garland and John Pearce from Leeds." From a personal experience of an episode of acute poisoning affecting four young men almost simultaneously they have drawn attention not only to the very varied neurological picture-which in their cases included very marked signs of parietal lobe disorder, visual impairment of cortical origin, transient deafness, epilepsy, and mental changes ranging from retardation to frank psychosis-but also to the capacity for these signs to vary quite dramatically and for the patient to relapse after apparent recovery. One-third of the New York State cases showing sequelae showed recovery and relapse, ${ }^{2}$ and other cases are on record in which after periods ranging from a few days to several weeks relapse occurred, leading to akinesia, Parkinsonism, and death. ${ }^{56}$ F. Plum and his colleagues $^{6}$ believed this occurred in patients who were got out of bed too soon. Though relapse may be associated with the extensive demyelination, in practice recovery even from the relapse is usually complete.

The mortality rate for carbon monoxide poisoning is based on figures which often exclude many accidental cases of mild degree. Add to this the rarity of permanent ill effects, and it becomes clear that if immediate effective treatment is available-perhaps by means of flying-squad teams-and patients are not allowed to return to activity too soon the prognosis is generally good. It is a paradox that many patients might not wish it so.

\section{Treatment of Gonorrhoea}

In 1958 F. R. Curtis and A. E. Wilkinson ${ }^{2}$ reported that some strains of gonococci showed in-vitro evidence of becoming less sensitive to penicillin and that the reduction in sensitivity correlated with a higher clinical relapse rate. As a result of this and other similar reports most physicians increased the routine dose to at least 600,000 units of procaine penicillin or penicillin in aluminium monostearate (P.A.M). The latter had the disadvantage of a slow rise and fall in serum concentration, whereas Curtis and Wilkinson had pointed out that the ideal penicillin preparation for the cure of gonorrhoea should have a rapid rise, high plateau of 1.0 unit per $\mathrm{ml}$. serum for 18-24 hours, and then a rapid fall in concentration. At about the same time $P$. Durel ${ }^{2}$ had reported clinical and laboratory evidence of an increasing percentage of streptomycin-resistant gonococci.

Thus it was thought that reports of penicillin-resistant strains from individual clinics should be amplified by a wider 